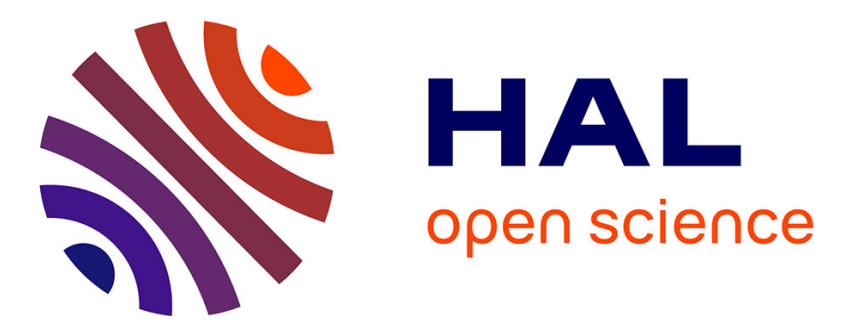

\title{
A Meta-Model-Based Procedure for Quantifying the On-Site Efficiency of Rockfall Barriers
}

\author{
Stéphane Lambert, David Toe, Alessio Mentani, Franck Bourrier
}

\section{To cite this version:}

Stéphane Lambert, David Toe, Alessio Mentani, Franck Bourrier. A Meta-Model-Based Procedure for Quantifying the On-Site Efficiency of Rockfall Barriers. Rock Mechanics and Rock Engineering, 2021, 54 (2), pp.487-500. 10.1007/s00603-020-02298-7 . hal-03129196

\section{HAL Id: hal-03129196 https://hal.science/hal-03129196}

Submitted on 16 Aug 2021

HAL is a multi-disciplinary open access archive for the deposit and dissemination of scientific research documents, whether they are published or not. The documents may come from teaching and research institutions in France or abroad, or from public or private research centers.
L'archive ouverte pluridisciplinaire HAL, est destinée au dépôt et à la diffusion de documents scientifiques de niveau recherche, publiés ou non, émanant des établissements d'enseignement et de recherche français ou étrangers, des laboratoires publics ou privés. 


\title{
A Meta-Model-Based Procedure for Quantifying the On-Site Efficiency of Rockfall Barriers
}

\author{
Stéphane Lambert ${ }^{1}$ (1) $\cdot$ David Toe $^{2} \cdot$ Alessio Mentani $^{3} \cdot$ Franck Bourrier $^{1}$
}

\begin{abstract}
This article proposes a procedure for developing tools to quantify the on-site efficiency of any rockfall barrier. This procedure relies on meta-modeling techniques to predict the barrier ability in arresting rock blocks, whatever their trajectory. For demonstration purpose, a specific low-energy barrier for which a finite element model was available is considered. The barrier response is simulated varying six parameters describing the rock block kinematics. Six different methods are used to create meta-models predicting the simulated barrier response. The ability of each method in creating meta-models with good prediction capacities is evaluated. Meta-models created utilizing the best methods are then used to quantify the efficiency of the barrier in arresting rock blocks in two real situations. These situations exhibit very similar $95 \%$ percentiles of the block passing height and kinetic energy but very different distributions for the other parameters describing the kinematics of the rock blocks. The predictions reveal that the barrier efficiency is extremely site-dependant. The discussion addresses the meta-models performance and highlights the benefits in using such meta-models for quantifying the barrier efficiency, in particular with respect to more classical barrier design approaches. Last, the proposed eight-step procedure for generating meta-models to be used in operational contexts is described.
\end{abstract}

Keywords Rockfall mitigation $\cdot$ Barrier $\cdot$ Meta-model $\cdot$ Rockfall simulations

\section{Introduction}

Various types of passive countermeasures are employed to protect roads, railways, living areas and buildings against rockfall. The definition of the best mitigation strategy and the design of protective structures depend on various parameters, including the block kinetic energy and the site topography in particular, and aim at reducing the hazard at the element at risk down to an acceptable value. This is in particular the case for barriers consisting in an interception structure hanged on post-supported cables. Barriers are the most widely used structures in this context and their variety has considerably increased since their first use in the 1970s.

Stéphane Lambert

stephane.lambert@inrae.fr

1 Univ. Grenoble Alpes, INRAE, ETNA, 38000 Grenoble, France

2 Univ. Grenoble Alpes, INRAE, LESSEM, 38000 Grenoble, France

3 Universitá di Bologna, Via Zamboni, 33, 40126 Bologna, Italy
In operational contexts, the selection of the appropriate barrier to install on a given site mainly relies on the results from tests conducted following the European Assessment Document (EAD) dealing with rockfall protection kits (EOTA 2018). This document describes a standardized conformance test procedure consisting in impacts on real-scale barriers with the aim of determining the barrier service and maximum energy levels (SEL and MEL, respectively). The MEL relates to the barrier impact strength and is here considered as the barrier reference capacity. For design purpose, the barrier reference capacity is often compared with the expected on-site rockfall kinetic energy (Peila and Ronco 2009). Nevertheless, this barrier reference capacity is related to a specific loading condition and has been shown to fail in traducing the global response of the barrier, in particular when considering the variety of on-site loading cases (Tran et al. 2013; Mentani et al. 2016; Toe et al. 2018; CastanonJano et al. 2018).

The ability of barriers in efficiently arresting rock blocks depends on their response to the various dynamic loadings associated with impacts under real conditions. By contrast with real-scale experiments, numerical models calibrated on 
appropriate experimental tests appear to be an efficient and cost-saving alternative to address the mechanical response of barriers. The efficiency of numerical models in simulating the barrier dynamic response has considerably increased over the past few years, so that the models can account for the numerous geometrical and material non linearities (Albaba et al. 2017; Buzzi et al. 2015; Castanon-Jano et al. 2018; Coulibaly et al. 2017, 2018, 2019; Dugelas et al. 2019; Escallon et al. 2015; Gentilini et al. 2013; Mentani et al. 2015, 2018; de Miranda et al. 2010; Tahmasbi et al. 2019; von Boetticher and Volkwein 2019; Xu et al. 2018; Yu et al. 2019b, a; Zhu et al. 2019). The predictive capacity of both finite element and discrete element numerical models has now reached a level of reliability allowing for their use as design improvement tools, and in particular in view of addressing the efficiency of barriers in reducing the hazard down to a targeted value, for a given site.

The main limitation in using numerical models to quantify the barrier on-site performance lies in the computation cost. Indeed, input data used for this purpose are issued from rockfall trajectory simulations which generally generate thousands of trajectories, all different from each other in terms of block kinematics at the barrier location. The time required for simulating the barrier response when considering all these cases is not affordable. Probabilistic reliability-based approaches were first considered for circumventing this limitation (Bourrier et al. 2015), before the use of meta-models was proposed (Mentani et al. 2016; Toe et al. 2018). In this context, meta-models can be defined as mathematical operators describing the response envelop of the barrier while considering a large number of variables. More precisely, one meta-model predicts one outcome associated with this response. Meta-models make it possible to obtain at nearly no computation cost a descriptor of the barrier response when considering the site-specific trajectories. Alternatively, meta-models may also be used to determine and quantify how the variability in some structural components influences the response of the barrier and affects its performances (Coulibaly et al. 2019; Dugelas 2019).

In their previous works, the authors developed a metamodel based on the support vector machine approach (SVM) to address the ability of the barrier in arresting the rock block and another one for estimating the reduction of the rock block velocity in case of structural failure (Toe et al. 2018). This work was conducted considering a low-energy barrier for which a finite element method (FEM) model was available (de Miranda et al. 2015). The authors have in particular shown that meta-modeling constitutes a powerful and efficient way for computing the failure probability of barriers. The advantage of a meta-model-based design over a design based on the barrier reference capacity was demonstrated and quantified. Nevertheless, the different impact conditions were considered equiprobable while their probability is extremely variable from one site to the other.

This study is dedicated to the development and evaluation of a new meta-model-based approach to integrate realistic impact loading conditions into the assessment of the onsite barrier efficiency in arresting blocks. The same barrier, FE model and parameters describing the kinematics of the blocks as in the previous study were considered. The metamodeling approach was used in view of predicting the barrier performance in terms of success in arresting rock blocks for any real site where the expected block kinetic energy is close to the barrier capacity. Two scenarios in similar conditions and based on a real configuration were defined in accordance with the barrier mechanical and geometrical characteristics. In both scenarios, the $95 \%$ rockfall kinetic energy percentile is close to the barrier reference capacity as determined from simulations of impacts in conditions following the requirements from the EAD. This approach corresponds to a design based on the maximum energy level (MEL) value issued from EAD tests.

This article is structured as follows. First, the barrier and its FEM model are presented. Then, the meta-model optimization strategy is presented. This strategy consists in considering six meta-model creation methods for creating meta-models based on $300 \mathrm{FE}$ model simulation results and in selecting the meta-model creation methods with higher performances. Then, the two scenarios are described, with a particular focus on the kinematics of the rock blocks. For demonstration purposes, the meta-models prediction is then evaluated considering 100 loading cases, which confirms the best method choice. Finally, the created meta-models are used for assessing the global barrier performance for both scenarios, showing the advantages and limitations of this approach. The discussion that follows addresses the metamodels performance, the benefits of using meta-models and proposes an eight-steps procedure for creating and using meta-models in operational contexts.

\section{Barrier Model}

\subsection{Barrier Description}

The barrier considered in this study is a low-energy structure of $3.2 \mathrm{~m}$ height that is commonly used to mitigate rockfall risk along roads and infrastructures. The structure consists of posts fixed to the ground that have the function to sustain the interception structure, which is primarily composed by horizontal cables of $12 \mathrm{~mm}$ in diameter and by a hexagonal net which prevent small blocks to pass through the barrier. The external posts are also secured by lateral cables of 18 $\mathrm{mm}$ diameter, which are connected to the ground (Fig. 1). 


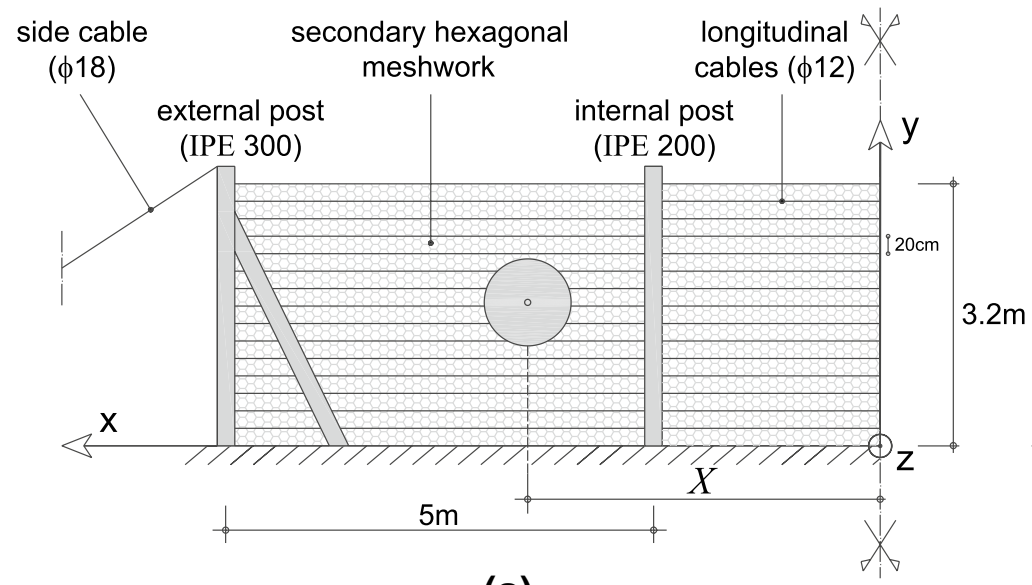

(a)

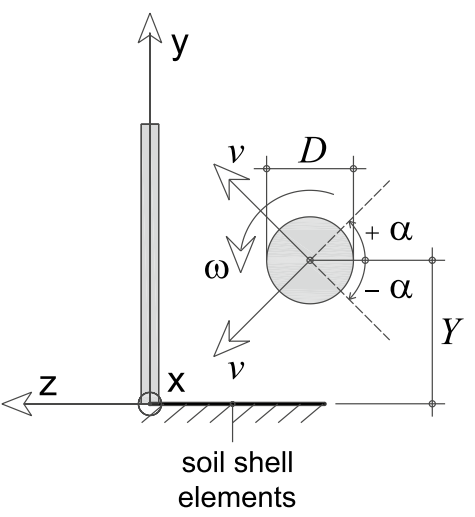

(b)

Fig. 1 Geometry and impact conditions for the cable net barrier: a back view and $\mathbf{b}$ side view. The origin and axes are also shown

\subsection{The FEM Model}

The barrier numerical model was developed with the FE commercial code Abaqus (Abaqus 2013). Following the EAD recommendations, the model was realized for a threespan barrier with posts spaced at $5 \mathrm{~m}$.

The posts were modeled with one-dimensional beam elements with an elastic-perfectly plastic constitutive model, while all other elements were modeled as elastoplastic cables or trusses that can harden in the plastic phase (de Miranda et al. 2015). A failure criterion was assigned to the net elements according to a suitable experimental investigation carried out on the net single elements and on net portions (Thoeni et al. 2013; Mentani et al. 2015). The posts were fully restrained at the base, while only displacements were constrained at the lateral anchorages. The sliding of the longitudinal cables through eyelets elements placed along the internal posts was modeled using connector elements between nodes existing in the FE code.

The impacting block was modeled as a spherical rigid body with a friction contact law assigned to the barrier elements with penalty friction coefficient of 0.4 (Escallòn et al. 2014; Mentani et al. 2016; Coulibaly et al. 2018). A small portion of the soil ground surface was also added uphill the barrier model to account for the possible block-soil contact prior or during the first phase of block impact (Fig. 1b), which occurred in few of the FE simulations described in Sect. 2.3. The soil surface was modeled with shell rigid elements and a friction-less law was assigned allowing no dissipation of the block kinetic energy.

\subsection{FE Simulations}

The barrier reference capacity was determined running simulations that followed the principles of the EAD. The test prescribed in this document consists in normal-to-thebarrier impacts in the center of a three-panel barrier by a projectile with translational velocity of at least $25 \mathrm{~m} / \mathrm{s}$ and no rotational velocity. Impacts were simulated considering these conditions. The block density was $2400 \mathrm{~kg} / \mathrm{m}^{3}$ and its volume was increased until barrier structural failure was reached (Toe et al. 2018). The maximum block mass for which the EAD requirements were fulfilled, was found equal to $640 \mathrm{~kg}$, yielding a reference capacity of $200 \mathrm{~kJ}$ for the cable net barrier. This threshold is a rounded value and is slightly less than the actual one. In view of building the meta-models, 300 simulations of impacts against the barrier were carried out by varying six input parameters describing the rock block incident kinematics just before impact: the block volume $V$; the block impact position on the barrier $X$ and $Y$; the incident angle of the impact $\alpha$; the translational velocity $v$; and the rotational velocity along the $\mathrm{x}$ axis $\omega$ (Fig. 1).

The trajectory deviation with respect to the line of maximum gradient (associated with biased trajectories) was not considered, making the meta-modeling approach directly compatible with $2 \mathrm{D}$ trajectory simulation tools. The rotational velocities with respect to the $\mathrm{z}$ and $\mathrm{y}$ axes were considered as having a limited influence on the barrier response and were thus not accounted for.

The values selected for these six parameters cover realistic ranges for natural rockfall (Table 1). Some ranges were adapted in order to account for the barrier characteristics. The maximum volume was identified based on FE simulations as the maximum size the barrier was able to sustain in quasi-static conditions. The ranges for parameters describing the impact position were set at the barrier dimensions. In addition, the minimum impact height (impact position along the $y$ axis) was set at the minimum block radius. The parameter values describing the impact conditions were randomly 
Table 1 Input parameters defining the loading conditions

\begin{tabular}{lll}
\hline Input parameter & Unit & Range min-max \\
\hline Translational velocity, $v$ & $\mathrm{~m} / \mathrm{s}$ & $5-30$ \\
Rotational velocity, $\omega$ & $\mathrm{rad} / \mathrm{s}$ & $0-35$ \\
Volume of the block, $V$ & $\mathrm{~m}^{3}$ & $0.03-2.14$ \\
Incident angle, $\alpha$ & $\mathrm{deg}$ & $-60-80$ \\
Impact position, $X$ & $\mathrm{~m}$ & $0-7.5$ \\
Impact position, $Y$ & $\mathrm{~m}$ & $0.15-3.2$ \\
\hline
\end{tabular}

sampled in these ranges to constitute the input parameters for the $300 \mathrm{FE}$ simulations. Starting with the sampling of the block volume, some non-relevant or impossible combinations with other parameters were excluded. The kinetic energy, referring to the block volume and its velocity, was limited to 1.5 times the barrier reference capacity. Indeed, limiting the number of cases leading to structural failure due to excessive kinetic energy improves the performance of the meta-model in the barrier intended operation domain (Toe et al. 2018). The impact height was checked to be higher than the block radius and to be less than the barrier height reduced by the block radius, to avoid direct impact on the ground surface and on the upper cable. Similarly, the impact position along the $x$ axis was compared to the block radius to avoid impact on the external post. As a result of all these restrictions, the distributions of some of the six parameters are not uniform (Fig. 2).

\section{Barrier meta-modeling}

\subsection{Meta-model creation}

The meta-model aims at predicting whether the barrier failed or succeeded in arresting the blocks. Similarly as in the previous study (Toe et al. 2018), failure may result from a structural failure (e.g., puncture of the mesh, rupture of a main cable, impact on a post) or from a block rolling over the structure (due to a block with high passing height having a high inclination upward trajectory). Basically, the metamodel allows determining the position of any loading case with respect to the boundary delineating the two domains associated, one to barrier failure and the other to success in arresting the block, in the $6 \mathrm{D}$ space defined by the calibration parameters.

In view of creating a robust and optimised meta-model, the strategy consisted in considering and comparing different meta-model creation methods. For that purpose, the choice was made to use the R package Caret, that embeds creation methods for hundreds of meta-models (Kuhn et al. 2018). Six meta-models creation methods were selected (Table 2) to illustrate the capacities of classical techniques extensively
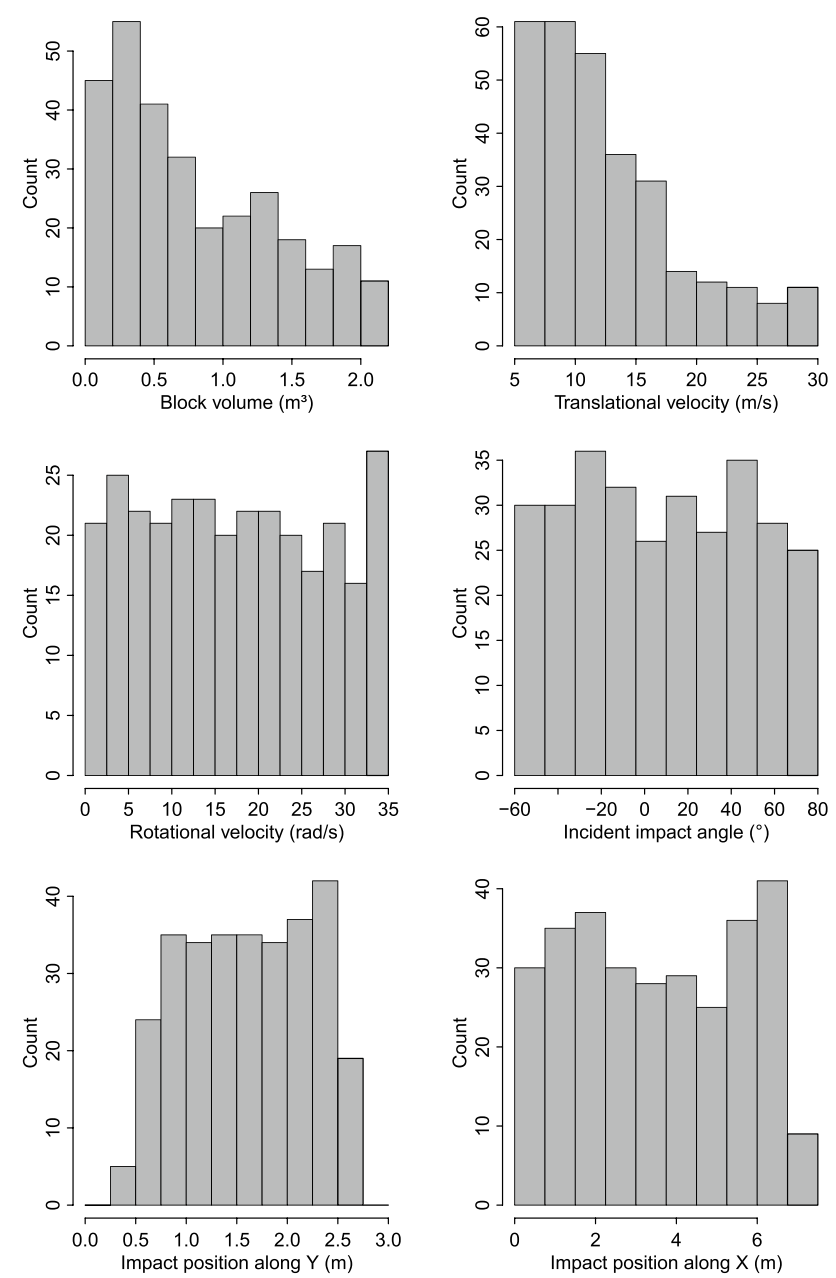

Fig. 2 Distributions of the input parameters values used for creating the meta-models

Table 2 Meta-model creation methods considered (see text for model name and reference)

\begin{tabular}{|c|c|c|}
\hline $\begin{array}{l}\text { Meta-Model } \\
\text { ID }\end{array}$ & $\begin{array}{l}\text { Method name in } \\
\text { the } \mathrm{R} \text { package }\end{array}$ & Method best fit parameters \\
\hline Metal & $x g b$ Dart & $\begin{array}{l}\text { nrounds }=150, \text { max_deph }=1 \text {, } \\
\text { eta }=0.4, \text { gamma }=0 \\
\text { subsample }=1, \text { colsam- } \\
\text { ple_bytree }=0.6, \text { rate_ } \\
\text { drop }=0.01 \\
\text { skp_drop }=0.95, \text { min_child_ } \\
\text { weight }=1\end{array}$ \\
\hline Meta2 & $f d a$ & degree $=1$, nprune $=14$ \\
\hline Meta3 & $r f$ & mytry $=2$ \\
\hline Meta4 & nnet & size $=5$, decay $=0$ \\
\hline Meta5 & svmradial & $\operatorname{sigma}=0.132, \mathrm{c}=1$ \\
\hline Meta6 & glmnet & alpha $=0.55$, lambda $=0.0258$ \\
\hline
\end{tabular}


used in the literature. The selected methods, adapted for creating binary class recognition meta-models, are :

- Metal : combination of regression trees (Rashmi and Gilad-Bachrach 2015);

- Meta2 : Flexible discriminant analysis (Hastie et al. 1994a);

- Meta3 : Random Forests (Breiman 2001),

- Meta4 : Neural Network (Ripley 1996; Venables and Ripley 2010);

- Meta5 : Support Vector Machines with Radial Basis Function Kernel (Vapnik 1995);

- Meta6 : Lasso and Elastic-Net Regularized Generalized Linear Models (Hastie et al. 1994b).

This set of methods focuses on widely used ones. No specific additional attempt was made to optimize this selection considering the tens of relevant-to-the-purpose methods available in the Caret package.

For each method, a meta-model was built using the 300 FE simulations. Comparison of the performance of the different meta-models was conducted in view of determining the best meta-modelling method for this barrier.

The creation of a meta-model started defining the values of the best fit parameters for the method. These parameters were those resulting in the highest predictive capacities. The number of method parameters depends on the method (Table 2) which can vary within predefined ranges. This phase was carried out using the functions of the Caret package available in $R$ software, including its train function (Kuhn et al. 2018).

The train function samples combinations of method parameters before assessing the meta-model predictive capacities for each combination. The number of combinations depends on the number of method parameters relevant to the method. For each combination of method parameters, the predictive capacity of the created meta-models was assessed using the so-called repeated $k$-fold cross-validation method (Bengio and Grandvalet 2004) with $\mathrm{k}=5$ and 25 repetitions.

In the repeated $k$-fold cross-validation approach used, a specific calibration procedure was repeated 25 times. This procedure consisted in, first, randomly split the 300 simulation results in five samples of 60 simulations. Then, five meta-models were created by calibration based on the five different combinations of four samples. The predictive capacity of each of the five calibrated meta-models was evaluated by comparison with the remaining sample and the percentage of good predictions $Q_{i}$ was calculated for each calibrated meta-model. In total, $125(25 * 5)$ calibrated meta-models and associated quality indicators $Q_{i}$ were obtained for each method and for each combination of tested parameters, which allowed calculating the mean
$Q\left(M_{M e t}\right)$ of $Q_{i}$ indicators. Finally, the method best fit parameters, leading to the highest value of $Q\left(M_{M e t}\right)$, were selected for each method (Table 2).

\subsection{Comparison of the Meta-Modeling Methods}

The comparison of the meta-modeling methods relies on $Q\left(M_{M e t}\right)_{B F}$, which is the value of $Q\left(M_{M e t}\right)$ computed considering the predictions by the meta-models created using the method best fit parameters only. $Q\left(M_{M e t}\right)_{B F}$ is the more straightforward indicator for comparing the different metamodeling methods and, eventually, selecting the best one. By contrast, $Q\left(M_{M e t}\right)$ refers to the method, as it evaluates the mean predictive capacities of several meta-models created using a given method and a given set of method parameters but considering different calibration dataset. The automatic choice of the method best fit parameters for each meta-modeling method was done using only this indicator because automatic selection processes are easier to set-up and more robust if based on a single indicator. However, this only indicator is not sufficient for meta-modeling methods comparison and selection purposes. It was, thus, complemented with two other indicators.

First, the standard deviation $\operatorname{st} d\left(Q_{i}\right)_{B F}$ of the $Q_{i}$ indicators calculated in the calibration process, for the method best fit parameters, can provide information on the variability of the predictive quality of the meta-models created using a same method and depending on the calibration data set used. This criterion, thus, ensures that all meta-models created considering the same method and same method parameters provide predictions of similar quality.

In addition, the correctness of the predictions by the meta-modelling methods may be assessed using the misclassification rates defined as follows. With reference to FE observations, the meta-model can provide bad (false, $F$ ) or good prediction (true, $T$ ). As described in Table 3, a good prediction is either positive $(T P)$ when barrier success $\left(B_{\text {Succ }}\right)$ is both predicted and simulated or negative $(T N)$ when barrier failure $\left(B_{F a i l}\right)$ is both predicted and simulated. Similarly, a false prediction is either positive $(F P)$ when barrier success is estimated instead of failure or negative $(F N)$ when barrier failure is estimated instead of success. Based on these definitions, two indicators can be used to discuss the performance of the meta-models: the false-negative rate $\left(F N_{r}=\frac{F N}{F N+T P}\right)$ and the false-posi-

Table 3 Definition of the cases used for computing the misclassification rates

\begin{tabular}{lll}
\hline FE observation & $\begin{array}{l}\text { Meta-model } \\
\text { prediction }\end{array}$ \\
\cline { 2 - 3 } & $B_{\text {Fail }}$ & $B_{\text {Succ }}$ \\
\hline$B_{\text {Fail }}$ & $T N$ & $F P$ \\
$B_{\text {Succ }}$ & $F N$ & $T P$ \\
\hline
\end{tabular}


tive rate $\left(F P_{r}=\frac{F P}{F P+T N}\right)$. In the context of this study, the false-positive rate $\left(F P_{r}=\frac{F P}{F P+T N}\right)$ is the most relevant criterion to use. Indeed, a high $F P_{r}$ value is associated with an overestimation of the barrier capacity by the metamodel which is detrimental when using the meta-model for design purpose.

The detailed analysis of mis-classification can be used to compare the meta-modeling methods. For that purpose, for each meta-modeling method, all the predictions done during the calibration phase using the method best fit parameters (60 predictions * 125 meta-model calibrations) were used to calculate the global percentage of false-positive predictions $F P_{G l o b a l, B F}$ and false-negative predictions $F N_{\text {Global,BF}}$. In particular, $F P_{\text {Global,BF }}$ provides information on the tendency of each meta-modeling method to overestimate the barrier capacity.

Table 4 presents the evaluation of the six meta-model creation methods. All methods result in relatively good predictive capacities with $Q\left(M_{M e t}\right)_{B F}$ values in a rather narrow range (70-77\% approx). Meta 1 and Meta 2 present slightly better results, with similar values. The $\operatorname{std}\left(Q_{i}\right)_{B F}$ obtained with these two methods is also good, as compared to the other methods, and in particular Meta 4 . By contrast with $Q\left(M_{M e t}\right)_{B F}, F P_{G l o b a l, B F}$ values exhibit an higher discrepancy. With respect to this criterion, Meta 1 and Meta 2 present better values than the four other methods, with an advantage for the latter. $F N_{\text {Global,BF}}$ values confirm that Meta 1 is the best, while Meta 2 is slightly less good. Nevertheless, this criterion is less important in our case, as mentioned before. In the end, Meta 1 and Meta 2 present better predictions, considering both criteria. As a consequence, these two methods will be preferred in the following.

Table 4 Evaluation of the six methods (all results in \%)

\begin{tabular}{lllll}
\hline Models & $Q\left(M_{\text {Met }}\right)_{B F}$ & $\operatorname{std}\left(Q_{i}\right)_{B F}$ & $F P_{\text {Global }, B F}$ & $F N_{\text {Global }, B F}$ \\
\hline Meta1 & 77.17 & 4.9 & 22.36 & 23.21 \\
Meta2 & 77.27 & 5.2 & 18.01 & 27.18 \\
Meta3 & 74.0 & 5.1 & 27.27 & 24.75 \\
Meta4 & 70.15 & 6.8 & 28.09 & 31.52 \\
Meta5 & 72.13 & 4.9 & 27.53 & 28.10 \\
Meta6 & 72.83 & 5.4 & 27.12 & 27.27 \\
\hline
\end{tabular}

\section{Meta-Model-Based Barrier Efficie y Assessment}

This section addresses the use of meta-models for predicting the barrier efficiency considering two real situations. In this aim, for each method a new meta-model was created using the entire set of $300 \mathrm{FE}$ simulations, considering the method best fit parameters (Table 2) and still using the Caret package available in $R$ software.

The considered scenarios are first described in details. Then, the predictions by the meta-models created using the six methods are compared considering a limited number of loading cases obtained from these scenarios. This comparison confirms the conclusion drawn at the end of the previous section. Finally, the barrier efficiency is quantified using Meta 1 and Meta2.

\subsection{Scenarios}

The approach was applied to a real-case site located in the 'Foret communal de Vaujany' in the French Alps where the element at risk to protect is a forest road. The slope is inclined at $38^{\circ}$ and covered with forest, this latter playing no significant role in the following scenarios. On this site, rockfalls are reactivated from small topographical outgrowths (Bourrier et al. 2015).

Considering this site, the two scenarios were defined in terms of block volume, release point, and traveling distance to the barrier in view of obtaining two different loading cases while being in accordance with the two main barrier characteristics. First, the rock block translation kinetic distributions of both scenarios should be such that the $95 \%$ percentile is less or equal to the reference barrier capacity (200 $\mathrm{kJ})$. This induces limits for the block velocity and its mass. Second, the rock block passing height should be such that the upper cable is not impacted: the barrier height reduced by the block radius is higher than the block passing height.

The two defined scenarios are similar in terrain characteristics (soil and global steepness) but differ in terms of local topography and distances between the release point and the barrier (Fig. 3). The two scenarios consider released blocks with a volume ranging uniformly from 0.1 to $1 \mathrm{~m}^{3}$.

In the first scenario (SCR1), the release area of the block is located at mid slope $(130 \mathrm{~m}$ in horizontal distance from the road in a snow avalanche corridor. The barrier is positioned on a little terrace $60 \mathrm{~m}$ in horizontal distance below the release area. In the second scenario (SCR2) the release area of the block is located $100 \mathrm{~m}$ in horizontal distance above the road and the barrier is located just above the road. By comparison with the first scenario, this scenario is prone to trajectory lateral deviation. 


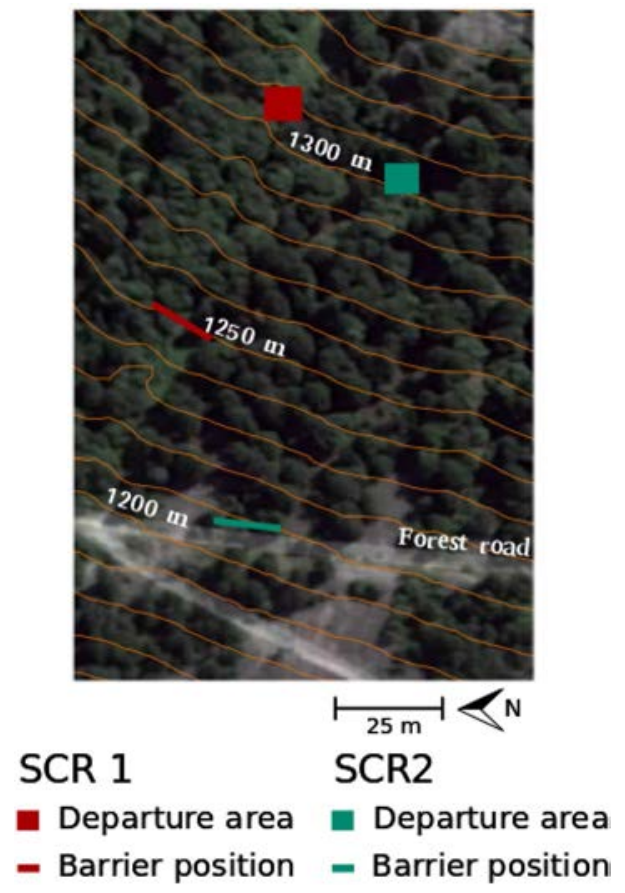

Fig. 3 View of the site, showing the two scenarios

Rockfall simulations were conducted using the 3D rockfall model RockyFor3D (RF3D) (Dorren 2015). RF3D is a model that simulates block trajectories on forested or nonforested slopes. The model simulates the propagation of spherical blocks along a slope modeled as a Digital Terrain Model (DTM) in raster format. The block propagation is modeled by a succession of free flights, impacts on the slope surface and impacts on trees. The rolling motion of the block is considered as a succession of rebounds and the sliding of the block over the slope surface is not taken into account, which is not a limitation in this context due to the slope steepness. The parameters governing the block rebound had been calibrated according to field measurement campaigns done on the same site, and presented in previous studies (Dorren et al. 2006; Bourrier et al. 2009, 2015).

10,000 blocks were released for each scenario. The initial falling height of the block was set to $0.5 \mathrm{~m}$ in the aim of initiating the block movement. The trajectories of the released blocks were characterized along two virtual lines in the digital terrain model. The two lines were located where rockfall barriers could be installed (Fig. 3). The six parameters describing the kinematics of the blocks (see Table 1) were measured along these lines.

\subsection{Blocks Kinematics at the Barriers Location}

Over the 10,000 rockfall simulations only 2677 blocks reach the barrier location for SCR 1 and 4712 blocks reach the barrier location for SCR2. This is due to the arrest of blocks
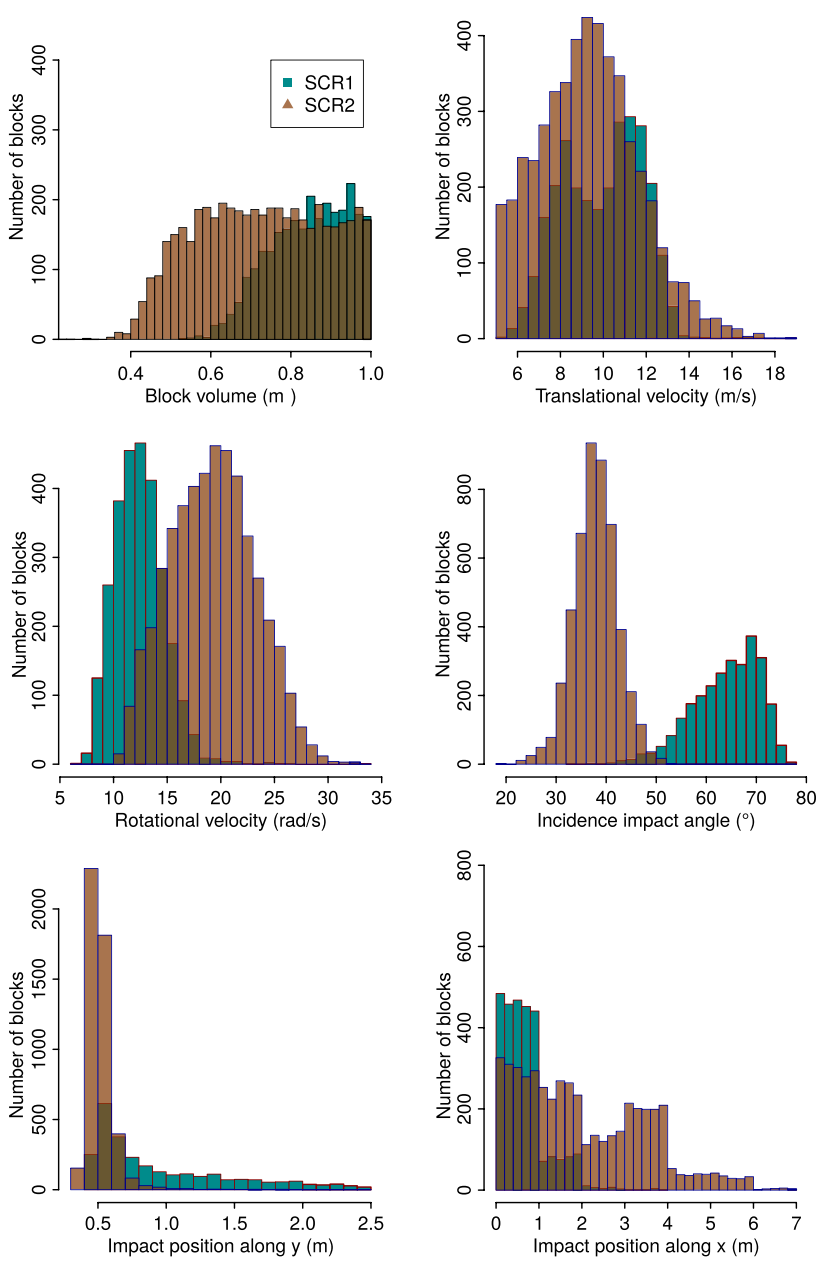

Fig. 4 Blocks kinematic parameters distribution at the barriers location for the two scenarios

before reaching the barrier, for SCR1 in particular, combined with blocks' lateral deviation for SCR2.

The distribution of the six parameters describing the kinematics of these blocks at the barrier locations are presented in Fig. 4. All values are within the parameter ranges considered for creating the meta-model (Table 1, Fig. 2). It is confirmed that $95 \%$ of the blocks reaching the barriers have an energy smaller than $200 \mathrm{~kJ}$.

These distributions show significant differences from one scenario to the other. The block volume distribution is more spread for SCR2 with volume ranging between 0.3 and 1 $\mathrm{m}^{3}$ compared to the range between 0.55 and $1 \mathrm{~m}^{3}$ for SCR1. In fact, smaller blocks stop before reaching the barrier in SCR1. This observation reveals that the volume distribution of the blocks reaching the barrier is not that in the release area, but should be considered as depending on the slope uphill the barrier, similarly as other kinematic parameters.

The block impact heights are smaller in SCR2 $(0-1 \mathrm{~m})$ compared to that in SCR1 (0-2.5 m). The incidence impact 
angle ranges between 20 and $40^{\circ}$ for SCR2 and between 40 and $80^{\circ}$ for SCR 1 .

In SCR2 rotational velocities are slightly larger (10 to 30 $\mathrm{rad} / \mathrm{s}$ ) compared to SCR1 (5-20 rad/s). The impact position along the $\mathrm{x}$ axis is more spread for SCR2 with block impact distributed from the center to the edge of the barrier. By contrast, impact positions are located around the center of the barrier for SCR1, and more precisely in the central panel. By comparison with other parameters, smaller differences are observed for the block translational velocity distributions.

These trajectory simulation results define the combinations of parameters describing the loading cases to consider for assessing the efficiency of the barrier for each scenario. It is noticeable that, as a consequence of these distributions, the loading cases associated with the two scenarios concentrate in part of the space defined by the six parameters and their ranges (Fig. 5). This figure also reveals that the two
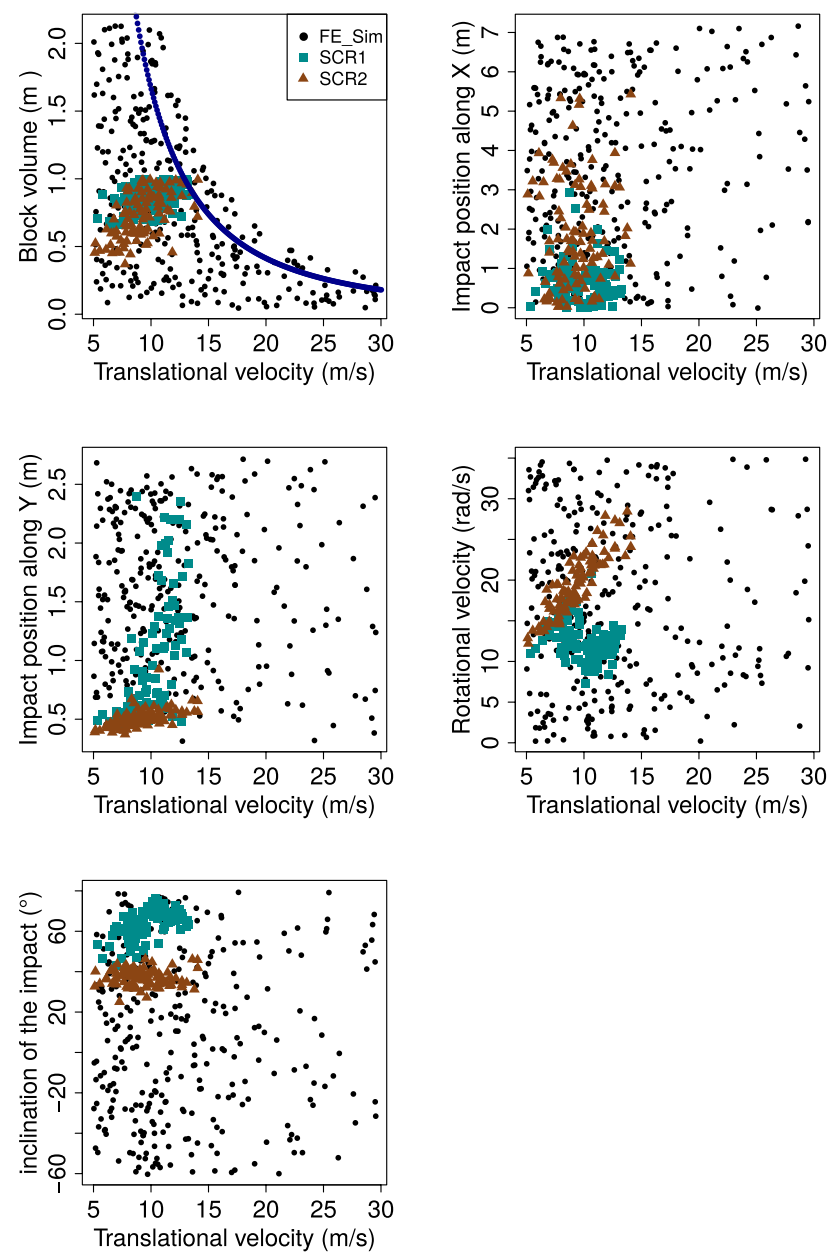

Fig. 5 Parameters describing the kinematics of the blocks at the barrier location. Significant difference in distribution are observed between SCR1, SCR2 and the values considered for running the FE simulations

scenarios differ one from the other in particular in terms of impact position along the $y$ axis, rotational velocity and trajectory inclination.

These differences stem from the differences in topographical and slope properties uphill the barriers. For example, the soil roughness is larger in SCR1, in particular favoring the arrest of smaller blocks before the barrier. In SCR2, the barrier is located just after a flat area, resulting in lower incidence impact angles.

\subsection{Evaluation of the Meta-Models Predictions}

Before considering the predicted barrier efficiency for the two scenarios, the relevance of the meta-modeling approach was addressed, in particular with the aim of confirming the choice of best method made in Sect. 3. In this aim, 100 rockfall loading cases were considered, by random sampling out of all trajectories of blocks reaching the barrier in scenarios one and two (2,677 and 4,712 blocks, respectively). The barrier response to these 100 loading cases was simulated using the FE model.

Table 5 and 6 show the comparison of the simulation results with the predictions by the different meta-models, ordered depending on the ratio of good predictions. The ranking slightly differs from one scenario to the other.

For SCR1, Meta2, Meta3 and Metal give the best results with ratios of good predictions as high as $94 \%, 85 \%$ and $86 \%$ respectively, and low $F P_{r}$ of $14.3 \%, 0 \%$ and $14.3 \%$, respectively. On the other side, Meta4, Meta5 and Meta6 are

Table 5 Ranking of the metamodels according to their predictive performance for SCR1 (all results in \%)

Table 6 Ranking of the metamodels according to their predictive performance for SCR2 (all results in \%)

\begin{tabular}{llll}
\hline Models & $\begin{array}{l}\text { Good } \\
\text { predic- } \\
\text { tions }\end{array}$ & $F P_{r}$ & $F N_{r}$ \\
\hline Meta2 & 94.0 & 14.3 & 5.4 \\
Meta3 & 85.0 & 0 & 16.0 \\
Meta1 & 86.0 & 14.3 & 14.0 \\
Meta4 & 95.0 & 71.4 & 0 \\
Meta6 & 93.0 & 100.0 & 0 \\
Meta5 & 93.0 & 100.0 & 0 \\
\hline
\end{tabular}

\begin{tabular}{llll}
\hline Models & $\begin{array}{l}\text { Good } \\
\text { predic- } \\
\text { tions }\end{array}$ & $F P_{r}$ & $F N_{r}$ \\
\hline Meta1 & 73.0 & 57.5 & 0 \\
Meta3 & 67.0 & 70.2 & 0 \\
Meta4 & 65.0 & 74.5 & 0 \\
Meta2 & 61.0 & 83.0 & 0 \\
Meta5 & 60.0 & 85.0 & 0 \\
Meta6 & 53.0 & 100 & 0 \\
\hline
\end{tabular}


confirmed to be clearly unable to predict the barrier response to these loading cases as giving $F P_{r}$ values are as high as $71.4,100$ and $100.0 \%$, respectively. For SCR2, the predictions are not as good. Metal gives the highest ratio of good predictions (73\%) and the lowest $F P_{r}(57 \%)$. The other metamodeling methods give $F P_{r}$ values above $70 \%$.

For SCR1, the number of failures observed from simulations, predicted by Meta 1 and by Meta 2 are 7,19 and 11, respectively. For SCR2, these values are 47, 20 and 8, respectively. This comparison suggests that Meta 2 is less efficient in predicting failures.

Two general trends emerge from these results. First, it shows that the meta-models, which appeared to be the most efficient after creation based on the sampling of parameters out of uniform distributions, are also the most efficient to predict the structure response when considering a limited number of site-specific loading cases. In particular, Metal and Meta 2 are confirmed to be the most appropriate metamodels for this barrier, suggesting that the meta-modeling strategy presented in Sect. 3 is efficient.

The second trend is that the global performance of the meta-model is not intrinsic to the model but also depends on the set of considered loading cases, and thus on the site. Even if Metal and Meta 2 are efficient for both scenarios, the difference between the predictions for the two suggests a significant influence of the distribution of the parameters describing the impact on the meta-model failure predictions. This is also the case for the four other meta-models.

These trends will be further discussed in Sect. 5.1.

\subsection{Quantification of the Barrier Efficiency}

The predictions for the two scenarios by meta-models Metal and Meta2 are synthesized in Fig. 6, where success and failure in arresting the blocks are represented using green and red symbols, respectively. The results are presented as a function of the block volume and translational velocity, as these constitute the basic parameters for describing a rockfall. The blue line corresponds to the barrier reference capacity of $200 \mathrm{~kJ}$.

For SCR1, the rounded ratio of predicted barrier success is $82 \%$ and $91 \%$ with Meta 1 and Meta2. For SCR2, these values are, respectively, $75 \%$ and $91 \%$. Meta1, thus, appears conservative for both scenarios and SCR2 exhibits an higher ratio of cases leading to failure. This is consistent with the the trend of an underestimation of the number of failures by Meta2 (Sect. 4.3).

It is relevant to highlight that many failure cases are observed below the barrier reference capacity, for both scenarios. Inversely, some barrier success are
Fig. 6 Predictions of the barrier response by Meta 1 and Meta 2 for the two scenarios

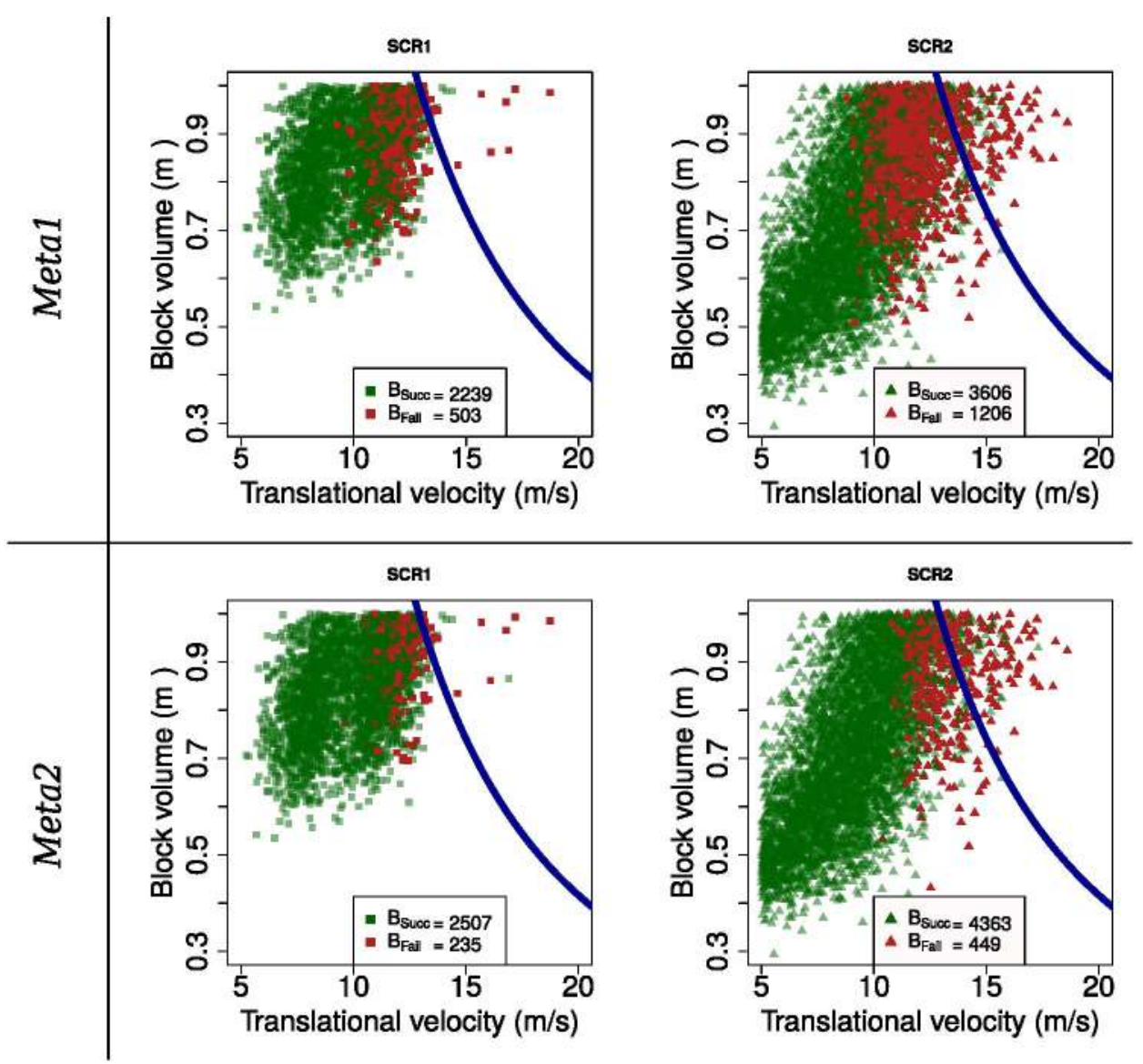


observed above the barrier reference capacity. More precisely, the predictions with Meta 1 reveal that $17.6 \%$ and $23 \%$ of the cases below the blue line lead to failure, for SCR1 and SCR2. Here again, Meta 2 seems to underestimate the number of failures, with ratios of $8.0 \%$ and 7.0 $\%$, respectively. Also, a significant part of these cases corresponds to block kinetic energies far below the barrier reference capacity. This confirms that an EAD-based design considering the MEL value would over-estimate the efficiency of the barrier in reducing the hazard down to the expected value.

The predictions with the two meta-models reveal two similar trends when considering the influence of other parameters. In SCR1, failures are associated with impacts in the upper part of the barrier (Fig. 7, left). This is not observed for SCR2, as impacts occurred below $1 \mathrm{~m}$ in height. The predictions for SCR2 reveal a strong dependence on the impact location position along the $\mathrm{x}$ axis (Fig. 7, right). In particular, a lower ratio of failure cases is observed at proximity of the post (x values in the $1-3 \mathrm{~m}$ range). This trend is not observed for SCR 1 because impacts concentrated in the barrier center.

\section{Discussion}

\subsection{Meta-Model Performance}

In this study, meta-models are used to predict the ability of a barrier in arresting rock blocks while considering six parameters describing the block kinematics. In this context, the performance of a meta-model is related to its ability in determining whether or not a loading case belongs to the domain associated with failure, in the 6D space formed by this six parameters. Among the wide array of available ones, six relevant-to-the-purpose methods were considered for creating six meta-models. Beyond the variability in method performance observed at the end of the creation process, two methods were selected as more efficient in predicting the barrier response. This choice was confirmed considering 100 loading cases sampled from each of the two scenarios considered, for what the meta-model results were compared with the outcome of FE simulations. Even though 100 loading cases may not be representative of the very large variety in loading cases, the results reveal that the global performance of the meta-model is site specific.

The parameter distributions considered for creating the meta-models are rather uniform, within wide ranges defined
Fig. 7 Barrier responses predicted with Meta1 and Meta2: as a function of the block translational velocity and impact position along the $y$ axis for SCR1 (left) and as a function of the block translational velocity and impact position along the $\mathrm{x}$ axis for SCR2 (right)

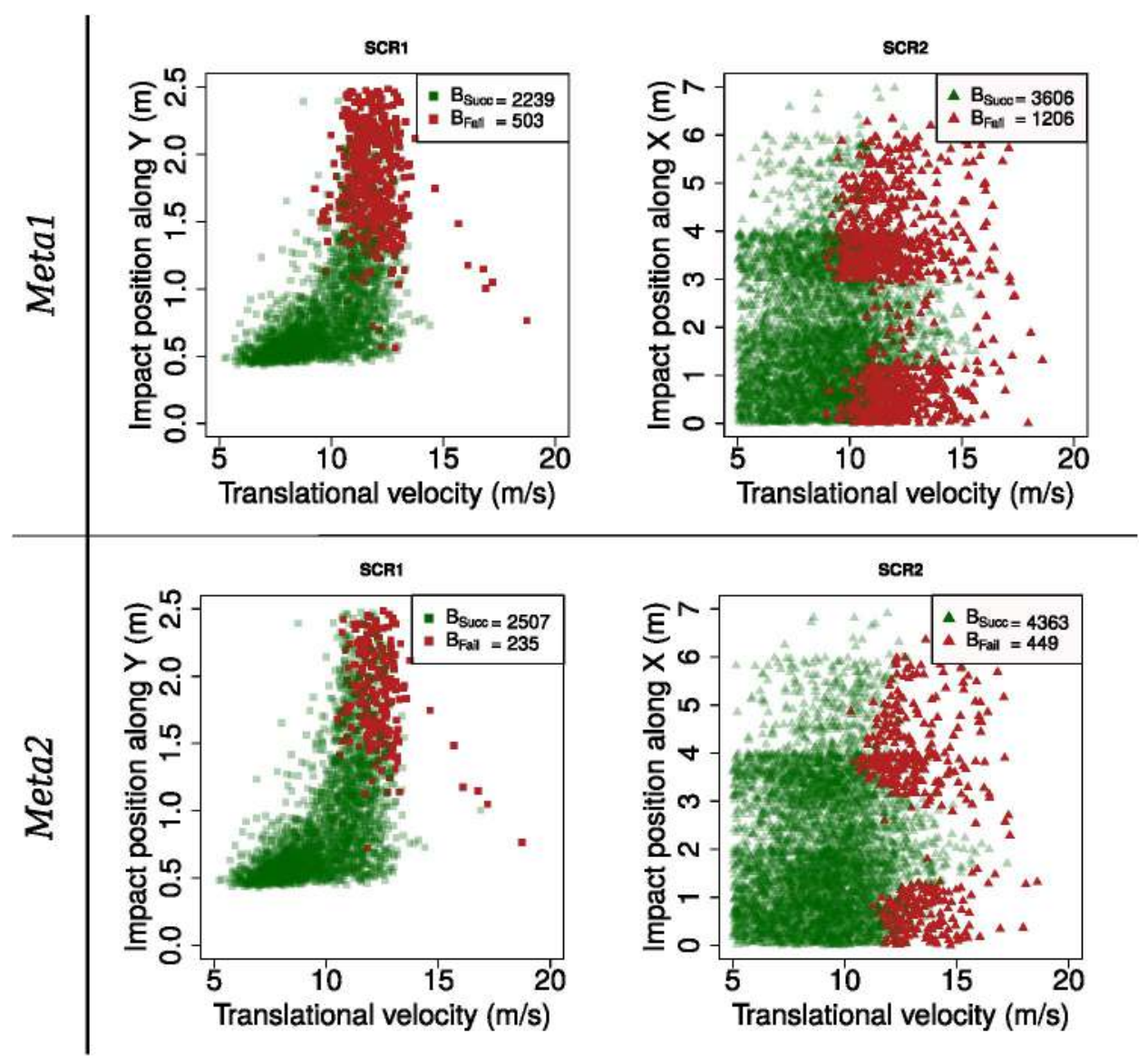


in accordance with the barrier mechanical and geometrical characteristics in particular. Each of the 300 combinations of six parameters has the same probability of occurrence. This drastically contrasts with real situations where loading cases may concentrate in part of the $6 \mathrm{D}$ space, thus with restricted parameters ranges and variable occurrence probability (Fig. 5). This is particularly critical when loading cases concentrate at the vicinity of the success/failure boundary because this is where predictions are more likely to be erroneous. By contrast, predictions are generally very good when the loading case is far enough from this boundary. The global performance of a meta-model is, thus, better when the parameter values and their combinations vary over wide ranges, than when loading cases concentrate close to the boundary. This is thought to explain the poorer prediction performance for SCR2 observed in Sect. 4.3 where the set of loading cases lead to a higher number of failures that the meta-models are less able to well predict.

More generally, reducing the dependence of the metamodel global performance on the distribution of loading cases requires improving the ability of the meta-model in closely fitting the failure envelope of the barrier in the $6 \mathrm{D}$ space. In other words, the local prediction error should remain equally small all along the failure envelope vicinity. This requirement in particular implies that the meta-model is able to account for the non-linearities in the barrier response. Non-linearity may in particular results from the location of the impact point with respect to some singular elements of the barrier (posts, cables). For example, Fig. 7 (right) suggests a reduction in failure cases associated with the presence of the post, at $x=2.5 \mathrm{~m}$. This trend is not confirmed by FEM simulations results presented in Fig. 8 where no change in the success/failure ratio is observed in the post vicinity. The meta-model is, thus, not precise enough in the region of the 6D space associated with the post and, in addition, overestimates the barrier efficiency. Non-linearities may also result from the evolution of the barrier response with the

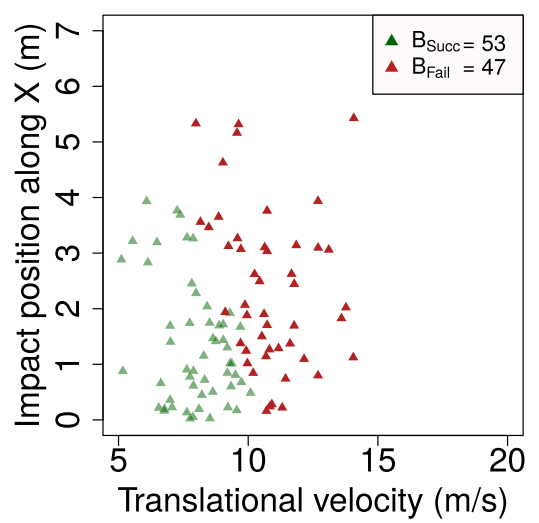

Fig. 8 FEM simulated barrier responses as a function of the block translational velocity and impact position along the $\mathrm{x}$ axis for SCR2 block velocity, as suggested by Bourrier et al. (2015). The ability of the meta-model in correctly predicting the barrier success whatever the distribution of loading cases could be improved by increasing the number of FE simulations used for creating the meta-model, in particular close to these nonlinearities. At this stage, 300 simulations were considered as a compromise between computation cost and meta-model reliability.

It is worth highlighting that the considered impact conditions do not consider biased trajectories nor rotational velocities around all block axes. This simplification is thought not to call into question the conclusions drawn and is assumed to be of negligible influence on the developed meta-models accuracy.

\subsection{Benefits Derived from a Barrier Meta-Model}

The results presented in Sect. 4.4 confirm that an EADbased barrier design fails in predicting the on-site barrier efficiency, when considering the MEL value. For both scenarios, the barrier reference capacity, determined considering the impact conditions prescribed in the EAD, is above the $95 \%$ percentile of the kinetic energy of the blocks reaching the barrier location. When exposed to on-site conditions, the real efficiency of the barrier appears to be much less, down to 82 and $75 \%$ of arrested blocks for SCR1 and SCR2, respectively. In other words, a non negligible number of impacts with kinetic energies less than the barrier reference capacity actually leads to barrier failure, due to very different impact conditions than that prescribed by the EAD. For example, Meta 1 predicts that $23 \%$ of the cases below the barrier reference capacity lead to failure for SCR2. The reason for the difference in global efficiency of the barrier, for both scenarios, is that the meta-model allows to account for the variety in impact conditions in the assessment of the barrier efficiency.

The proposed meta-modeling approach relies on a set of 300 simulations of the mechanical response of the barrier, performed using FEM in this case. The literature proposes more and more FEM and DEM numerical models for rockfall barriers, considering various barrier technologies. Some of these models have been shown to be relatively computationally inexpensive, for instance with computation times of a few minutes for simulating the impact response of a real barrier (e.g., 2, 5 and $17 \mathrm{~min}$ in Mentani et al. (2018), Coulibaly et al. (2019) and von Boetticher and Volkwein (2019)). Meta-model creation tools are now widely available through user-friendly softwares. Creating a meta-model is affordable in terms of computation time, once a numerical model for the considered barrier is available. This requires very limited time and effort, in particular by contrast with that required to develop and validate a numerical model for a specific barrier. The cost for creating a meta-model 
is even more negligible considering the fact that the same meta-model for a given barrier can be employed for any site, any configuration. Meta-models make it possible to compute the on-site efficiency of barriers in arresting the blocks and consequently in reducing the hazard down to the targeted value. It will lead to a significant improvement of quantitative rockfall hazard assessment considering protective barriers (Corominas et al. 2005; Agliardi et al. 2009). It allows a much more reliable barrier efficiency assessment than considering the maximum energy level obtained based on the EAD prescriptions. Conversely, for a given site, it would allow defining the optimum barrier meeting the hazard reduction requirement, accounting for the site-specific blocks trajectories. More precisely, it would be possible to quantify and compare the efficiency of different barriers adapted for the considered site providing a large set of meta-model is available. This seems to be a reasonable and enthusiastic perspective considering the increasing number of developed and validated numerical models of commercially available barriers. More generally, meta-models could be used in view of proposing safety coefficients to be considered in design recommendations and standards. For example, such meta-models could allow proposing reduction factors to be applied on the EAD-based reference capacity so that the designed barrier actually plays its hazard reduction role. There is indeed a strong demand for adapting the principles of the Eurocodes for the design of rockfall barriers, which recently motivated research works for proposing safety coefficients (Vagnon et al. 2017; De Biagi et al. 2020).

\subsection{Proposed Procedure for Creating a Barrier Meta-Model for Operational Purpose}

Based on this study, it is possible to propose a procedure for creating rockfall barrier meta-models to be used in operational contexts when coupled with rockfall trajectory simulation tools.

Developing such a meta-model requires a numerical model simulating the barrier mechanical response to impact. This model should be properly calibrated and validated, based on real-scale impact experiments. Whatever the modeling approach (e.g., FEM, DEM), a low computation cost model would be preferable in view of computing the thousands of simulations required to create the meta-model.

This procedure consists in the eight successive steps described below:

1. Using the numerical model, determine the barrier reference capacity in accordance with the EAD requirements (normal and centred impact on a 3-panel structure, impact velocity not less than $25 \mathrm{~m} / \mathrm{s}$ ).

2. Determine the parameters describing the rock block kinematics with an influence on the barrier response. These will serve as input parameters for the meta-model. At least consider the six parameters indicated in Table 1. In principle, the proposed procedure allows considering a higher number of parameters.

3. Define the ranges for the input parameters. In this purpose, consider the values indicated in Table 1, and adjust the ranges of the coordinates of the impact location and the maximum block volume to the barrier dimensions.

4. Choose a set of meta-model creation methods adapted for binary class recognition, for example among those available in some tools such as R. In this article, six creation methods were considered.

5. Generate a set of combinations of the considered input parameters, by sampling within the pre-defined ranges. During this phase, some combinations should be avoided. For instance, combinations resulting in a block kinetic energy higher than 1.5 the barrier reference capacity should be excluded. The impact position of a block with a given volume (and thus radius) should be such that neither the upper cable nor the external posts are impacted. The number of required combinations in particular depends on the number of parameters required for creating the meta-models. In this study, 300 combinations were considered for six parameters. This number of combinations revealed sufficient to create meta-models with good predictive capacities for the considered structure and number of input parameters.

6. Simulate the barrier impact response considering the loading cases defined by this combinations set.

7. Assess the performance of the meta-model creation methods using the simulation results. The authors suggest using the user-friendly Caret package available in $R$, as it allows conducting all these tasks conveniently, including the determination of the method best fit parameters. After comparing their respective failure/ success predictions, select the best method(s). If several methods exhibit very similar predictive capacities, it is recommended to consider all these for the following steps. If none of the created meta-models exhibits satisfactory predictive capacities, consider another set of creation methods (step 4) or increase the number of simulations (step 5).

8. For each selected method and considering its best fit parameters, create a meta-model using the whole set of numerical simulation results.

The created meta-model(s) may be used to quantify the barrier efficiency for any site where the blocks passing height, volume and velocity are in accordance with the barrier characteristics. In this purpose, the blocks kinematic parameters along the projected position of the barrier should be used to serve as input data for the meta-model(s). Note that, at 
present and to the authors knowledge, no trajectory simulation tool allows direct implementation or coupling with a meta-model. In case several meta-models are used, it is recommended to retain the less favorable prediction in terms of barrier success.

\subsection{Conclusion}

This paper tackles the challenging and critical issue of the real efficiency of barriers in arresting rock blocks when considering site-specific blocks trajectories. An optimized meta-model-based procedure is proposed and applied for quantifying the efficiency of a specific barrier considering two realistic situations.

The proposed procedure relies on the creation of a metamodel specific to each barrier, which can further be applied to any site where the considered barrier is assumed to be adapted. The meta-model predicts the ability of the barrier in arresting the blocks, while considering six parameters describing the kinematics of the blocks. The creation of such a meta-model reveals not resources demanding as compared to the development and validation of the barrier numerical model required for creating the meta-model.

The procedure optimization relates to two crucial points. First, the parameter ranges considered to create the metamodel are optimized considering the barrier characteristics, in the aim of improving the prediction quality of the metamodel in the intended barrier operation domain. Second, the best meta-models are selected out of a set of several ones, created considering different methods.

Two main conclusions are drawn when using the barrier meta-model for two scenarios where the barrier is a priori expected to be efficient. First, the real efficiency of the barrier is extremely dependant on the rockfall scenario. Second, an efficiency assessment based on the barrier efficiency as obtained following the principles of the EAD is far too optimistic. For instance, in this study, up to $23 \%$ of the impact cases below the barrier reference capacity lead to barrier failure in arresting the blocks. Both conclusions bring to light the importance of the loading conditions on the barrier response, which strongly depends on the distribution of the different parameters describing the blocks trajectory.

The ability of meta-models in predicting the on-site barrier efficiency at a low computation cost makes it a powerful and reliable approach in view of improving the quantification of the hazard reduction resulting from the building of a barrier. A promising perspective could be to define an optimized strategy for improving the global performance of the predictions by the meta-models. This could be achieved by introducing more meta-modelling approaches in the process and by increasing the number of simulations used for creating the meta-models, focusing on the domain, in the $6 \mathrm{D}$ space formed by the kinematic parameters, where the response of the barrier shows non-linearities and where the meta-model prediction error is higher.

Acknowledgements FE modelling was conducted within the framework of a DICAM research fellowship, which activity was carried out under the main supervision of Prof. Guido Gottardi, who is therefore sincerely acknowledged.

Authors contribution The respective contributions of the authors are: SL wrote the paper, contributed to the developments and analyses done in the research work, in particular in the domains of rockfall protection structures and rockfall hazard. DT performed the numerical simulations related with the creation of the meta-models and with rockfall hazard assessment, produced the figures and contributed to the development and analysis of the research work and to the writing of the paper. AM performed the FE simulations required for creating the meta-models and contributed to the writing of the paper. FB provided the initial ideas of this research work, supervised the developments and analyses done in the research work, and wrote part of the paper.

\section{References}

Abaqus (2013) Abaqus Analysis User's Manual. Version 6.11

Agliardi F, Crosta GB, Frattini P (2009) Integrating rockfall risk assessment and countermeasure design by $3 \mathrm{~d}$ modelling techniques. Nat Hazards Earth Syst Sci 9(4):1059-1073

Albaba A, Lambert S, Kneib F, Chareyre B, Nicot F (2017) Dem modeling of a flexible barrier impacted by a dry granular flow. Rock Mech Rock Eng 50(11):3029-3048

Bengio Y, Grandvalet Y (2004) No unbiased estimator of the variance of k-fold cross-validation. J Mach Learn Res 5:1089-1105

Bourrier F, Dorren L, Nicot F, Berger F, Darve F (2009) Toward objective rockfall trajectory simulation using a stochastic impact model. Geomorphology 110(3):68-79

Bourrier F, Lambert S, Baroth J (2015) A reliability-based approach for the design of rockfall protection fences. Rock Mech Rock Eng 48(1):247-259

Breiman L (2001) Random forests. Mach Learn 45(1):5-32

Buzzi O, Leonarduzzi E, Krummenacher B, Volkwein A, Giacomini A (2015) Performance of high strength rock fall meshes: effect of block size and mesh geometry. Rock Mech Rock Eng 49:1221-1231

Castanon-Jano L, Blanco-Fernandez E, Castro-Fresno D, Ferreño D (2018) Use of explicit fem models for the structural and parametrical analysis of rockfall protection barriers. Eng Struct 166:212-226

Corominas J, Copons R, Moya J, Vilaplana JM, Altimir J, Amigó J (2005) Quantitative assessment of the residual risk in a rockfall protected area. Landslides 2(4):343-357

Coulibaly J, Chanut M-A, Lambert S, Nicot F (2018) Sliding cable modeling: an attempt at a unified formulation. Int J Solids Struct 130-131:1-10

Coulibaly, J., Chanut, M.-A., Lambert, S., and Nicot, F. (2019). Toward a generic computational approach for flexible rockfall barrier modeling. Rock Mech Rock Eng

Coulibaly JB, Chanut M, Lambert S, Nicot F (2017) Nonlinear discrete mechanical model of steel rings. J Eng Mech 143(9)

De Biagi V, Marchelli M, Peila D (2020) Reliability analysis and partial safety factors approach for rockfall protection structures. Eng Struct 213:110553

de Miranda S, Gentilini C, Gottardi G, Govoni L, Mentani A, Ubertini F (2015) Virtual testing of existing semi-rigid rockfall protection barriers. Eng Struct 85:83-94 
de Miranda S, Gentilini C, Gottardi G, Govoni L, Ubertini F (2010) A simple model to simulate the full-scale behaviour of falling rock protection barriers. In: Proceedings of the 7th International conference on physical modelling in geotechnics-ICPMG, volume 2, pp 103-108, Tokyo, Japan

Dorren L (2015) Rockyfor3d (v5.2) revealed. transparent description of the complete $3 \mathrm{~d}$ rockfall model. Technical report, ecoris Q

Dorren L, Berger F, Putters US (2006) Real-size experiments and 3-D simulation of rockfall on forested and non-forested slopes. Nat Hazards Earth Syst Sci 6(1):145-153

Dugelas L (2019) Probabilistic strategies applied to discrete numerical modeling : the case of rockfall flexible fences (in french). $\mathrm{PhD}$ thesis, Université Grenoble Alpes, Grenoble

Dugelas L, Coulibaly J, Bourrier F, Lambert S, Chanut M, Olmedo I, Nicot F (2019) Assessment of the predictive capabilities of discrete element models for flexible rockfall barriers. Int J Impact Eng

EOTA (2018) Falling rock protections kits. ead 340059-00-0106/c 417/07. technical report

Escallon J, Boetticher V, Wendeler C, Chatzi E, Bartelt P (2015) Mechanics of chain-link wire nets with loose connections. Eng Struct 101:68-87

Escallòn JP, Wendeler C, Chatzi E, Bartelt P (2014) Parameter identification of rockfall protection barrier components through an inverse formulation. Eng Struct 77:1-16

Gentilini C, Gottardi G, Govoni L, Mentani A, Ubertini F (2013) Design of falling rock protection barriers using numerical models. Eng Struct 50:96-106

Hastie T, Tibshirani R, Buja A (1994a) Flexible discriminant analysis by optimal scoring. J Am Stat Assoc 89(428):1255-1270

Hastie T, Tibshirani R, Buja A (1994b) Flexible discriminant analysis by optimal scoring. J Am Stat Assoc 89(428):1255-1270

Kuhn M, Wing J, Weston S, Williams A, Keefer C, Engelhardt A, Cooper T, Mayer Z, Kenkel B, Benesty M, Lescarbeau R, Ziem Z, Scrucca L, Tang Y, Candan C, Hunt T (2018) caret: Classification and Regression Training. $\mathrm{R}$ package version 6.0-81

Mentani A, Giacomini A, Buzzi O, Govoni L, Gottardi G, Fityus S (2015) Numerical modelling of a low-energy rockfall barrier: new insight into the bullet effect. Rock Mech Rock Eng 49(4):1247-1262

Mentani A, Govoni L, Giacomini A, Buzzi O (2018) An equivalent continuum approach to efficiently model the response of steel wire meshes to rockfall impacts. Rock Mech Rock Eng 49(51):2825-2838

Mentani A, Govoni L, Gottardi G, Lambert S, Bourrier F, Toe D (2016) A new approach to evaluate the effectiveness of rockfall barriers. Proc Eng 158:398-403

Peila D, Ronco C (2009) Technical note: design of rockfall net fences and the new etag 027 european guideline. Nat Hazards Earth Syst Sci 9(4):1291-1298
Rashmi KV, Gilad-Bachrach R (2015) DART: dropouts meet multiple additive regression trees. arXiv:1505.01866

Ripley BD (1996) Pattern recognition and neural networks. Cambridge University Press, Cambridge

Tahmasbi S, Giacomini A, Wendeler C, Buzzi O (2019) On the computational efficiency of the hybrid approach in numerical simulation of rockall flexible chain-link mesh. Rock Mech Rock Eng 52:3849-3866

Thoeni K, Lambert C, Giacomini A, Sloan SW (2013) Discrete modelling of hexagonal wire meshes with a stochastically distorted contact model. Comput Geotech 49:158-169

Toe D, Mentani A, Govoni L, Bourrier F, Gottardi G, Lambert S (2018) Introducing meta-models for a more efficient hazard mitigation strategy with rockfall protection barriers. Rock Mech Rock Eng 51(4):1097-1109

Tran P, Maegawa K, Fukada S (2013) Experiments and dynamic finite element analysis of a wire-rope rockfall protective fence. Rock Mech Rock Eng 46:1183-1198

Vagnon F, Ferrero A, Umili G, Segalini A (2017) A factor strength approach for the design of rock fall and debris flow barriers. Geotech Geol Eng 35:2663-2675

Vapnik V (1995) The nature of statistical learning theory. Springer, New York

Venables WN, Ripley BD (2010) Modern applied statistics with S. Springer, New York

von Boetticher A, Volkwein A (2019) Numerical modelling of chain-link steel wire nets with discrete elements. Can Geotech J 56(3):398-419

Xu H, Gentilini C, Yu Z, Qi X, Zhao S (2018) An energy allocation based design approach for flexible rockfall protection barriers. Eng Struct 173:831-852

Yu, Z., Liu, C., Guo, L., Zhao, L., and Zhao, S. (2019a). Nonlinear numerical modeling of the wire-ring net for flexible barriers. Shock and Vibration

Yu ZX, Zhao L, Liu YP, Zhao SC, Xu H, Chan SL (2019b) Studies on flexible rockfall barriers for failure modes, mechanisms and design strategies: a case study of western china. Landslides $16: 347-362$

Zhu Z-H, Yin J-H, Qin J-Q, Tan D-Y (2019) A new discrete element model for simulating a flexible ring net barrier under rockfall impact comparing with large-scale physical model test data. Comput Geotech 116:103208

Publisher's Note Springer Nature remains neutral with regard to jurisdictional claims in published maps and institutional affiliations. 